\title{
NUMERICAL SIMULATION OF OIL DISPERSION AFTER AN ACCIDENTAL SUBSEA BLOWOUT IN THE GULF OF PATRAS
}

\author{
Dimitrios-Periklis A. Giannoulis ${ }^{1 *}$, Nikolaos A. Avgerinos ${ }^{1}$, Dionissios P. Margaris ${ }^{1}$ \\ ${ }^{1}$ University of Patras, Mechanical Engineering and Aeronautics Department, Fluid Mechanics Laboratory, \\ Patras, Greece
}

\begin{abstract}
:
In recent years, there has been a growing interest in the exploitation of hydrocarbon deposits within the Greek territory, in the Aegean and the Ionian Sea. This increasing interest comes along with the possibility of an oil spill event of devastating impact on the environment, the marine life and the economic activity of the nearby coastal areas. In this context, the present work simulates the trajectory of leaking oil in the event of an accidental subsea blowout in the Gulf of Patras, in order to predict the underwater dispersion, to estimate the rise time needed for the underwater oil spill to reach the sea surface as well as the distance of this position from the accident location. Numerical simulations were performed using ANSYS Fluent software and the proposed numerical methods were validated against small-scale experimental results. Two cases involving oil leakage in the presence of transverse sea currents are examined, one for crude oil and one for chemically dispersed oil. Results for the rise times, the rise and horizontal migration velocities of the oil plume, as well as the distances traveled horizontally and vertically are presented.
\end{abstract}

ARTICLE HISTORY

Received: 13.12 .2020$.

Accepted: 17.03.2021.

Available: 31.03.2021.

\section{KEYWORDS}

ANSYS Fluent, dispersants, leaking oil, numerical simulation, subsea blowout

\section{INTRODUCTION}

Offshore oil production has been growing internationally in recent decades due to increased energy needs, thanks to the modern technologies that allow the exploitation of deposits at greater depths. This, combined with the complex environment in the drilling areas, increases the possibility of an accident leading to an oil spill due to an explosion or leak in subsea pipelines, with incalculable environmental and socio-economic impact.

In recent years, the use of computational fluid dynamics (CFD) methods to simulate underwater leakage events has become widespread. Options vary, depending on the use of Euler [1, 2] or Lagrange $[3,4]$ approach models and the comparison between experimental and numerical results [5]. However, due to the nature of the flow and the breakdown of the initial oil jet into small bubbles, the Euler approach requires a computational grid with cells smaller in size than the diameter of the bubbles simulated. As a result, there are limitations in implementation of realworld geometries due to the millions of cells required, a fact that dramatically increases the computational power and time required for the numerical simulations.

Recently, there has been a strong interest in the exploitation of hydrocarbon deposits within the Greek territory, in the Aegean and the Ionian Sea. However, while such a possibility could significantly enhance our country's role in the energy sector, we should not overlook the fact that possible oil spills can have devastating effects on the environment, marine life and economic activity in coastal areas. In this context, the need for a tool that can generate reliable predictions assisting first 
responders and other involved entities in emergency situations is obvious.

Makatounis et al. [6] presented a methodology for the contamination probability estimation in the Gulf of Patras and the associated environmental impacts on the wider area while Giannoulis and Margaris [7] examined the applicability of a system designed to remove leaking oil from ship wrecks, in offshore well blowouts.

In the present study, the authors intend to present a numerical simulation of the oil leak trajectory in the event of a subsea accident in the Gulf of Patras, using a reliable and time efficient computational model. The licensed area, Fig. 1, is $1892 \mathrm{~km}^{2}$ and has an average depth of $158 \mathrm{~m}$ while the examined depth is equal to $130 \mathrm{~m}[6,8]$. The originality of the work lies in the fact that for the first time, results for the subsea trajectory of an oil leak in the Gulf of Patras are presented, thus making another step forward towards understanding the requirements to addressing the adverse effects of a subsea accident in this area. This work provides substantial information on the prediction of the oil spread in the subsea area, the determination of the location in the sea surface where an oil slick is expected to be developed, and the estimation of the minimum response time to deal with such accidental events.

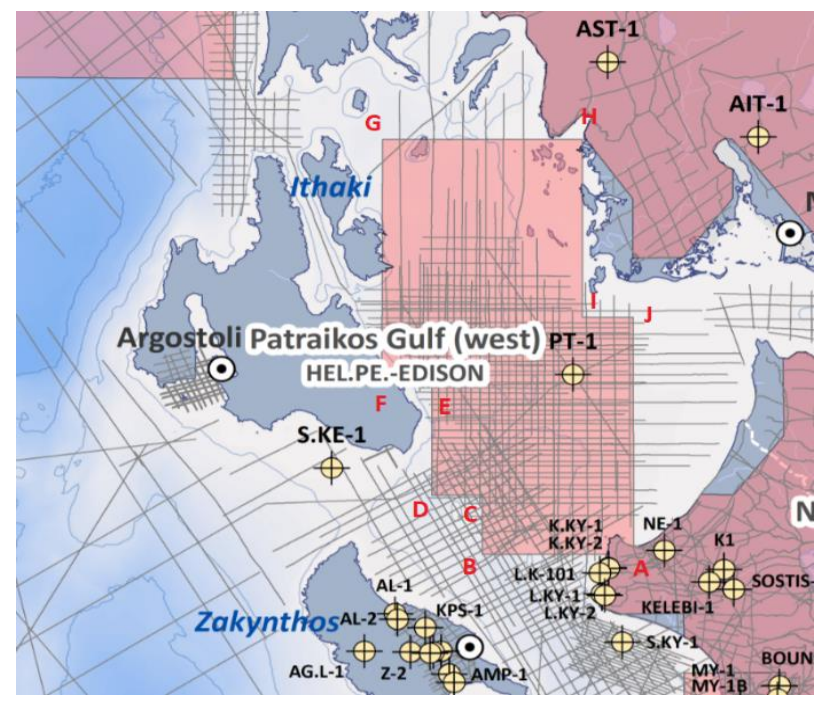

Fig. 1. Patraikos Gulf block (Property of HHRM S.A.) [9]

The proposed methodology can be used, with the appropriate modifications, in other cases of leaks at any point of extraction, being part of the Strategic Environmental Assessment plan, contributing to the organization of the timely response in the case of an accident.

In Section 2 the governing equations used in the numerical simulation, a comparison with experimental data to verify the computational model set-up, the model limitations and the grid independence study are presented. Section 3 introduces the methods used in the parametric study for the area of interest while in Section 4 the numerical results are presented and analyzed. Finally, Section 5 includes the main conclusions.

\section{COMPUTATIONAL MODEL}

\subsection{Numerical methods}

The geometries and the meshes were developed in the commercial software Gambit 2.2.30, which is a CFD pre-processor, appropriate for geometry and mesh generation [10] while the numerical investigations were conducted using the ANSYS Fluent 16.0 software [11].

A two equations turbulence model, standard $k$ $\varepsilon$, was used to address the turbulence in the flow field due to oil release and dispersion.

For the multiphase flow modeling, the EulerianLagrangian Discrete Phase Model (DPM) is employed, where the discrete phase can be simulated, and their trajectories can be computed by the particle force balance in a Lagrangian reference frame. DPM functionality is based on the assumption that the particles volume fraction is sufficient low, i.e. $10-12 \%$ based on the grid cell, thus having no effect to the continuous phase. This assumption suggests that a coarser grid is required for the computational task.

SIMPLE algorithm was used for the pressurevelocity coupling and PRESTO interpolation scheme was used for pressure. Momentum, turbulent kinetic energy and turbulent dissipation rate discretization schemes were second order, for precision issues. The Virtual Mass Force was enabled in the DPM model, to take into account the additional drag force on the droplets in case of possible acceleration, while the Random Walk Model is also activated in order to calculate the effect of instantaneous turbulent velocity fluctuations on the droplet trajectories. For the accuracy of the solution, a value of $10^{-6}$ was used for the residual terms. The time step was fixed at $0.05 \mathrm{~s}$.

\subsection{Governing equations}

Mass and momentum equations address the seawater movement, its effect on the escaping oil and the subsequent dispersion in the sea environment. The general equation of mass conservation is as follows: 


$$
\frac{\partial \rho}{\partial t}+\nabla \cdot(\rho u)=0
$$

Where $\rho$ is the density, $\boldsymbol{u}$ the velocity vector defined as $\boldsymbol{u}=u \boldsymbol{i}+\boldsymbol{v} \boldsymbol{j}+\boldsymbol{w} \boldsymbol{k}$, and $\nabla$ the operator expressed as $\nabla=i \frac{\partial}{\partial x}+j \frac{\partial}{\partial y}+k \frac{\partial}{\partial z}$.

The momentum conservation is expressed as:

$$
\frac{\partial(\rho u)}{\partial t}+\nabla \cdot(\rho u u)=-\nabla p+\nabla \cdot(\bar{\tau})+\rho g+F
$$

where $p$ is the static pressure, $g$ is the gravitational body force, $F$ the external body forces, i.e. interactions with the dispersed phase, and $\tau$ is the Newtonian fluid stress tensor given by:

$$
\bar{\tau}=\mu\left[\left(\nabla u+\nabla u^{T}\right)-\frac{2}{3} \nabla \cdot u I\right]
$$

where $\mu$ is the molecular viscosity and $I$ is the unit tensor. The volume dilation effect is enclosed in the second term of the right-hand side of the equation.

Regarding the standard $k-\varepsilon$ turbulence model, the first transport equation addresses turbulent kinetic energy $k$ and the second one the turbulent dissipation $(\varepsilon)$ :

$$
\begin{gathered}
\frac{\partial(\rho k)}{\partial t}+\frac{\partial}{\partial x_{i}}\left(\rho k u_{i}\right)= \\
\frac{\partial}{\partial x_{j}}\left[\left(\mu+\frac{\mu_{t}}{\sigma_{k}}\right) \frac{\partial k}{\partial x_{j}}\right]+G_{k}+G_{b}-\rho \varepsilon-Y_{M}+S_{k} \\
\frac{\partial(\rho \varepsilon)}{\partial t}+\frac{\partial}{\partial x_{i}}\left(\rho k u_{i}\right)= \\
\frac{\partial}{\partial x_{j}}\left[\left(\mu+\frac{\mu_{t}}{\sigma_{\varepsilon}}\right) \frac{\partial \varepsilon}{\partial x_{j}}\right]+C_{1 \varepsilon} \frac{\varepsilon}{k}\left(G_{3 k}+C_{3 \varepsilon} G_{b}\right)-C_{2 \varepsilon} \rho \frac{\varepsilon^{2}}{k}+S_{\varepsilon}
\end{gathered}
$$

where $G_{b}$ is the k generation due to buoyancy, and $G_{k}$ due to mean velocity gradients. The fluctuating dilatation in compressible turbulence to the overall dissipation rate is expressed by $Y_{M}$. The turbulent Prandtl numbers for $k$ and $\varepsilon$ are $\sigma_{k}$ and $\sigma_{\varepsilon}$, respectively. Model constants are as follows, $C_{1 \varepsilon}=$ 1.44, $C_{2 \varepsilon}=1.92, C_{\mu}=0.09, \sigma_{k}=1.0$, and $\sigma_{\varepsilon}=1.3$.

The interrelation between the particle inertia and the acting forces in the DPM model is expressed by the particle force balance given by the following system of equations:

$$
\begin{gathered}
\frac{d u_{p}}{d t}=F_{D}\left(u-u_{p}\right)+\frac{g\left(\rho_{p}-\rho\right)}{\rho_{p}}+F \\
F_{D}=\left(18 \cdot \mu \cdot C_{D} \cdot \operatorname{Re}\right) /\left(24 \cdot \rho_{p} \cdot d_{p}^{2}\right)
\end{gathered}
$$

$$
\operatorname{Re}=\rho_{l} \cdot d_{p} \cdot\left|u_{p}-u_{l}\right| / \mu_{l}
$$

where $F$ is the particle acceleration term incorporating gravity and drag effect, $u$ is the ambient fluid velocity, $u_{p}$ is the particle velocity, $d_{p}$ is the particle diameter, $R e$ is the Reynolds number, and $C_{D}$ is the drag force coefficient.

\subsection{Effects of natural gas presence}

The presence of natural gas in the leaking mixture, affects the oil bubble diameter distribution and the developed buoyancy force on the plume [12]. In a previous work, results of the coalescence procedure of methane bubbles under high pressure conditions were reported shading light in the hydrodynamic behaviour of the leading and trailing bubbles [13]. As such, in order to take into consideration these effects, the first step is to adjust the oil exit velocity at the leaking point, to take into account the gas volume fraction, as presented below:

$$
U_{\text {exit }}=U_{o i l} /(1-n)^{1 / 2}
$$

Then, a second adjustment is made to the exit velocity, to account for the increased buoyancy:

$$
U_{\text {exit }}^{\prime}=U_{\text {exit }}\left(1+F r^{-1}\right)
$$

The Froude number is expressed by:

$$
F r=U_{e x i t} /\left(g^{\prime} \cdot D\right)^{1 / 2}
$$

where

$$
g^{\prime}=g\left[\rho_{\text {water }}-\rho_{\text {oil }}(1-n)\right] / \rho_{\text {water }}
$$

The adjusted exit velocity $U^{\prime}$ exit is used to calculate the Weber and Reynolds numbers in the leaking point and finally the volume median droplet diameter, as follows:

$$
\left(d_{50} / D\right)=A \cdot W e^{-3 / 5}\left[1+B \cdot V i \cdot\left(d_{50} / D\right)^{1 / 3}\right]^{3 / 5}
$$

where $A$ and $B$ are empirical coefficients, $D$ is the leaking point diameter and $V i=W e / R e$.

The procedure for obtaining the oil bubble diameter distributions used in this study, with the above methodology, is analytically described in [12].

\subsection{Model validation}

Part of the tuning process of the computational model, is to validate it through the comparison of the results obtained by the numerical simulation of 
relative experiments available in literature, as it is not always feasible to obtain experimental results from full-scale experiments, for the real-world subsea areas of interest. For this reason, published data of the experimental work of Engebretsen et al. [14] are considered, regarding gas (air) release in a water-filled rectangular tank.

The above mentioned experimental set-up domain was modelled: a rectangular tank with dimensions $6 \mathrm{~m} \cdot 9 \mathrm{~m} \cdot 7 \mathrm{~m}$. The gradient adaption curvature method was enabled in order to achieve mesh refinement based on the DPM concentration. The final mesh consists of 238,529 hexahedral cells, with 80 cells along the plume centre, as specified for similar applications [3]. The initial mesh generation and its refinement are depicted in Fig. 2 and 3. The wall boundary conditions are selected for all the geometry surfaces except the upper surface where pressure outlet is assigned.

Three cases were examined, for air volume flow rates of $83 \mathrm{l} / \mathrm{s}, 170 \mathrm{l} / \mathrm{s}$ and $750 \mathrm{l} / \mathrm{s}$, respectively. The continuous phase was water, and the discrete phase was air in the form of bubbles, injected from the bottom of the tank from 100 point sources located in a diameter of $0.34 \mathrm{~m}$. The air bubble size was approximated by a Rosin-Rammler distribution with minimum and maximum diameters of $0.001 \mathrm{~m}$ and $0.01 \mathrm{~m}$ respectively and a value of 1.8 for the spread parameter was selected. Default ANSYS Fluent values were utilized for water and air properties.

The initial values for turbulent kinetic energy and turbulent dissipation were equal to $0.007 \mathrm{~m}^{2} / \mathrm{s}^{2}$ and $0.001 \mathrm{~m}^{2} / \mathrm{s}^{3}$ respectively [15].

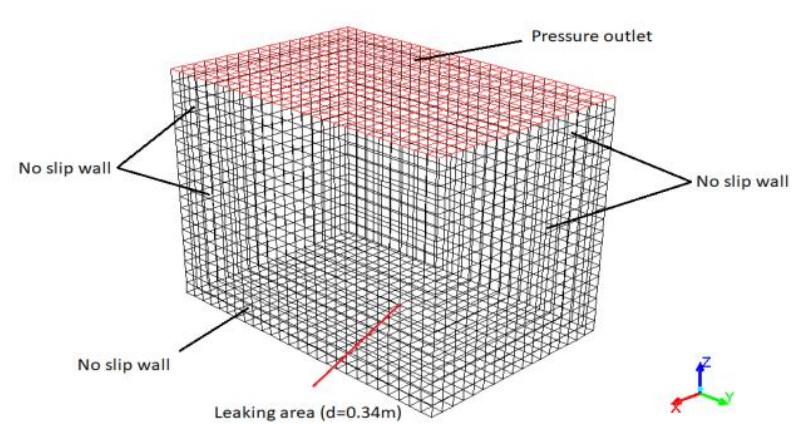

Fig. 2. Computational domain for the model validation

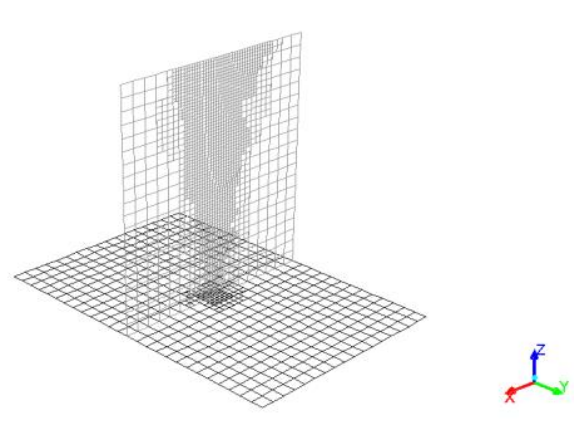

Fig. 3. Characteristic representation of the mesh refinement in the computational domain mid-plane

The simulation results are presented in Fig. 4, 5 and 6 and in Table 1. The comparison of the numerical predictions with the published experimental data is based on the bubble rise time for all flow rates and the radial distribution of the water induced velocity for the middle flow rate (170 l/s).

It is obtained from the numerical results that there is good agreement with the experimental ones, for both the rise times and the water velocity profiles for all three cases, as shown in Table 1. The rise times are slightly under-predicted for the first two cases (1.7\% and $3.1 \%$ respectively) and slightly over-predicted (8.1\%) for the third one. Next, the centre water velocity profiles presented in Fig. 6 . It can be observed that the centre water velocities are slightly overestimated but across the plume radius and right outside the plume, the experimental and simulation results match well.

Table 1. Rise time comparison

\begin{tabular}{|c|c|c|c|}
\hline $\mathrm{Q}(\mathrm{I} / \mathrm{s})$ & $\begin{array}{c}\text { Rise time (s) } \\
\exp \end{array}$ & $\begin{array}{c}\text { Rise time (s) } \\
\text { sim }\end{array}$ & Error \% \\
\hline 83 & 6 & 5.9 & 1.7 \\
\hline 170 & 4.8 & 4.65 & 3.1 \\
\hline 750 & 3.1 & 3.35 & 8.1 \\
\hline
\end{tabular}
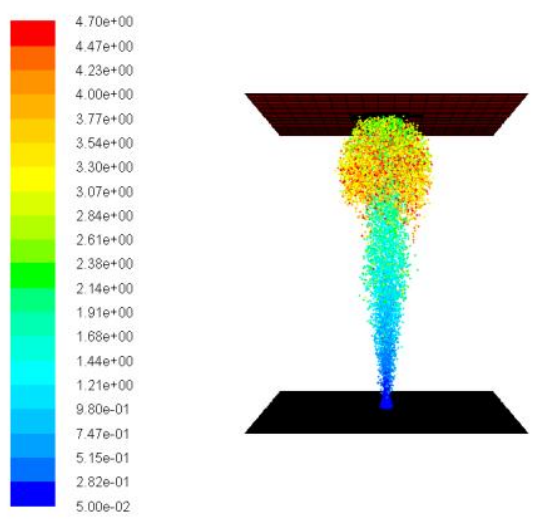

Fig. 4. Air bubble traces coloured by rise time (s) $(170 \mathrm{l} / \mathrm{s})$ 

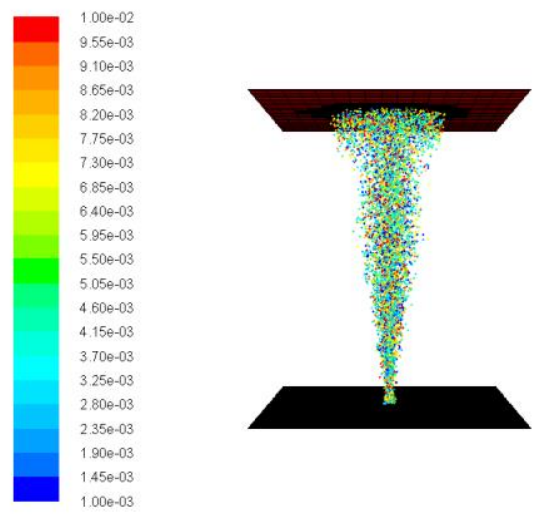

Fig. 5. Air bubble traces coloured by bubble diameter (m) after $6 \mathrm{~s}(170 \mathrm{l} / \mathrm{s})$

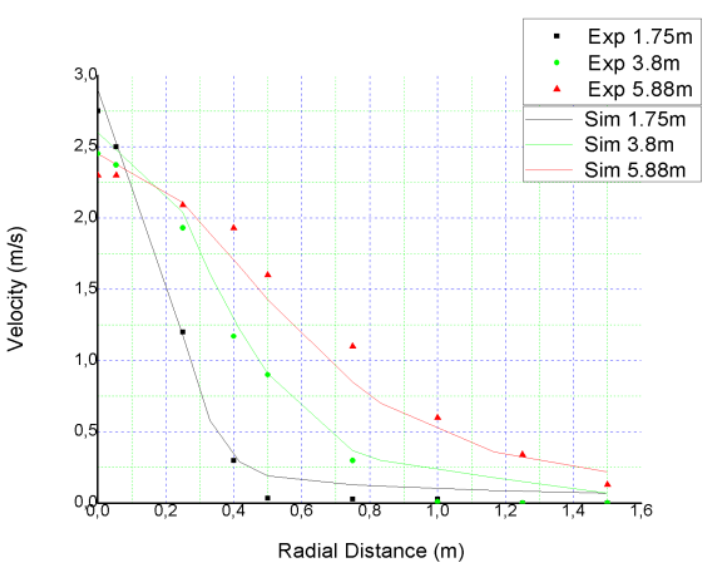

Fig. 6. Water velocity radial distribution, 3 elevations $(170 \mathrm{l} / \mathrm{s})$

The small differences in the above results arise due to the isotropic turbulent viscosity assumption of the $k-\varepsilon$ and the Random Walk models as well as the unavoidable violations of the particle volume fraction limit of $10-12 \%$ in some grid cells.

As a general conclusion from the comparison between the model predictions with the experimental results, it can be noted that the agreement between the experimental and simulation results is very satisfying meaning that the CFD model could faithfully reproduce the flow field of the experimental study, especially in terms of the rise time which is considered a crucial parameter in determining the available response time in case of an accident.

\subsection{Model limitations}

Albeit the very good agreement between the computational predictions and the experimental results regarding the plume trajectory, upscaling from the experimental small-scale set-up to the large-scale field of oil and gas exploitation applications is a very challenging process. The difficulty stems from the fact that there are no insitu measurements of the hydrodynamic parameters of the plume, such as the size distribution of the oil droplets or the gas bubbles, the rise time of the plume head, and the horizontal deflection of the smaller droplets by the sea current.

Bearing in mind the said simulation challenges, the present study is seeking for a holistic yet reliable and accurate approach to this problem. In this sense, the scope is to present a time effective and accurate CFD model to address the urgent demands arising when a deep-water oil spill occurs. In this context, the above presented numerical methods and physical parameters will be adopted.

\subsection{Mesh independency study}

A mesh independency study was carried out in order to select the appropriate cell dimensions for the computational domain of our analysis which is a rectangular region of $130 \mathrm{~m}$ height (z-axis), $300 \mathrm{~m}$ length (x-axis) and $300 \mathrm{~m}$ width (y-axis). Three different meshes were generated, consisting of 11,700 (Mesh 1), 42,320 (Mesh 2, Fig. 7) and 93,600 (Mesh 3) hexahedral cells and as the gradient adaption curvature method was enabled in order to achieve mesh refinement based on the DPM concentration, the final meshes contained 29,830, 94,498 (Fig. 12) and 195,765 respectively when the first oil bubble reached the sea surface. The initial cell width in each mesh was $10 \mathrm{~m}, 6.5 \mathrm{~m}$ and $5 \mathrm{~m}$ downsized to $2.5 \mathrm{~m}, 1.625 \mathrm{~m}$ and $1.25 \mathrm{~m}$ respectively after the mesh refinement. The cell number increased by 262\% from Mesh 1 to Mesh 2 and by $121 \%$ from Mesh 2 to Mesh 3, for the initial mesh and by $217 \%$ and $107 \%$ respectively for the refined mesh.

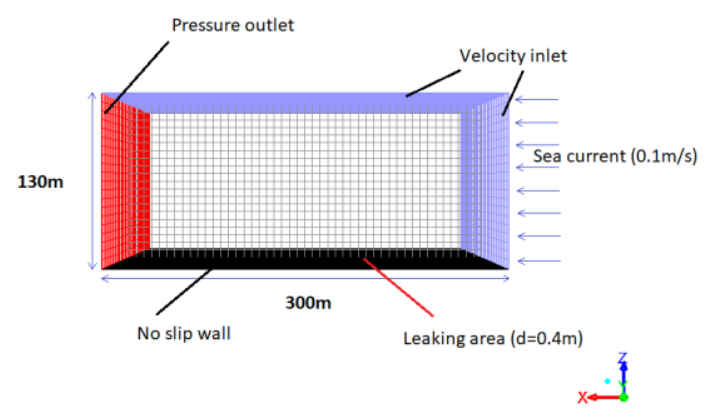

Fig. 7. Computational domain 
The boundary conditions utilized were velocity inlet for the upstream vertical surface, the side vertical surfaces of the computational domain, and the sea surface. Pressure outlet was used for the downstream vertical surface of the domain. No-slip wall boundary condition was selected for the bottom surface of the domain.

The oil characteristics used are that of the Oseberg blend, with $832 \mathrm{~kg} / \mathrm{m}^{3}$ density and $0.05 \mathrm{~Pa} \cdot \mathrm{s}$ dynamic viscosity $[6,12]$. After the appropriate void fraction adjustments, as described previously, the oil flow rate and the bubble diameter distribution were estimated. The oil volume flow rate was $0.11 \mathrm{~m}^{3} / \mathrm{s}$ and the gas volume fraction was $50 \%$. The oil bubble size was approximated by a Rosin-Rammler distribution with minimum and maximum diameter of $0.00305 \mathrm{~m}$ and $0.01885 \mathrm{~m}$ respectively, a mean diameter of $0.0077 \mathrm{~m}$, based on a bubble volume fraction above $3 \%$, and a value of 1.8 for the spread parameter [12]. As far as the sea current is concerned, the velocity value for the boundary condition was $0.1 \mathrm{~m} / \mathrm{s}$ [8].

The initial values of the turbulent kinetic energy and the turbulent dissipation, $0.00457 \mathrm{~m}^{2} / \mathrm{s}^{2}$ and $0.0091 \mathrm{~m}^{2} / \mathrm{s}^{3}$ respectively, due to the fact that the seawater is not stationary. However, these values are not expected to have any substantial effect on the results, due to the longer flow time compared with the validated model [3].

The comparison between the computational results for all the aforementioned meshes is made for the oil droplet rise time, the vertical and horizontal bubble velocities as well as for the rising heights and the horizontal migration distances during the drifting process.

Table 2. Comparison of the rise time for the mesh independency study

\begin{tabular}{|c|c|}
\hline Mesh & Rise time $(\mathrm{s})$ \\
\hline 1 & 352 \\
\hline 2 & 351 \\
\hline 3 & 351 \\
\hline
\end{tabular}

The rise time, as presented in Table 2, is decreasing with increasing cell number (or decreasing cell dimensions) from Mesh 1 to Mesh 2 and is stable in Mesh 3. As such, it is obvious that there is no further improvement in the rise time despite the increasing computational cost due to the additional mesh refinement. The same conclusion is obtained by the results of the horizontal migration distance and the bubble velocities, presented in Fig. 8 to 11.
The maximum difference for the vertical bubble velocities is $0.5 \%$ from Mesh 1 to Mesh 2 and $0.25 \%$ from Mesh 2 to Mesh 3 and for the horizontal migration distances, is $3.6 \%$ and $0.68 \%$ respectively. It is noted therefore that the differences for the rise time and the vertical velocity are similar. However for a more accurate prediction of the horizontal distance it seems that a finer mesh than Mesh 1 is needed. Similar differences were obtained for the horizontal velocities and the rising height.

The Mesh 2 is more appropriate candidate for the computational domain since the final cell size after the refinement is $1 / 80$ of the domain depth under examination, which is in agreement with findings of previous published work for similar applications [3]. This hypothesis is also supported by the findings of the mesh independency study presented in Table 2.

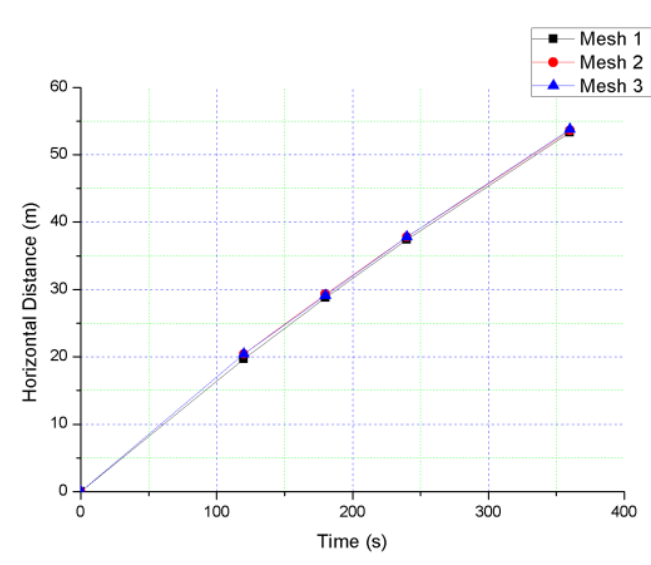

Fig. 8. Mesh independency study: Horizontal migration distance comparison

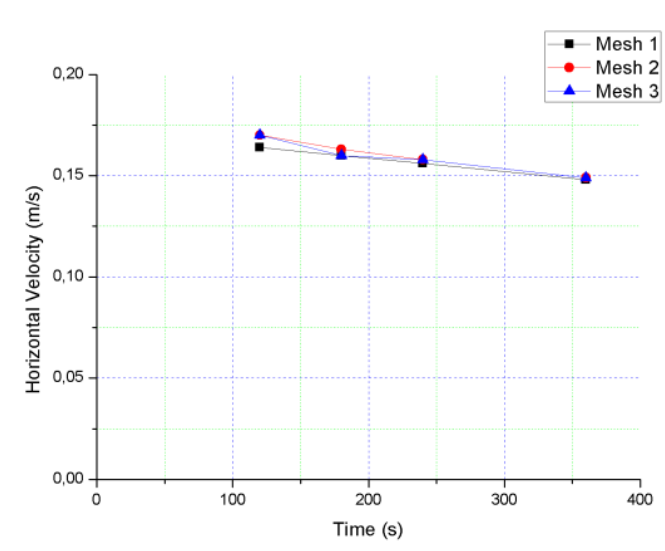

Fig. 9. Mesh independency study: Bubble horizontal velocity comparison 


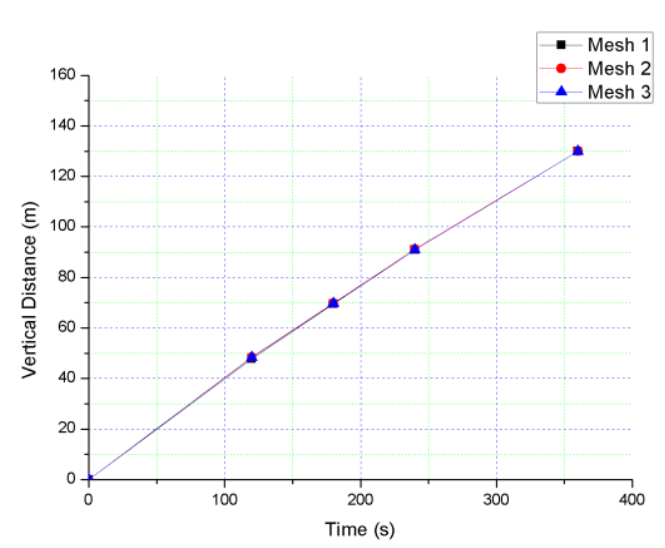

Fig. 10. Mesh independency study: Rising height comparison

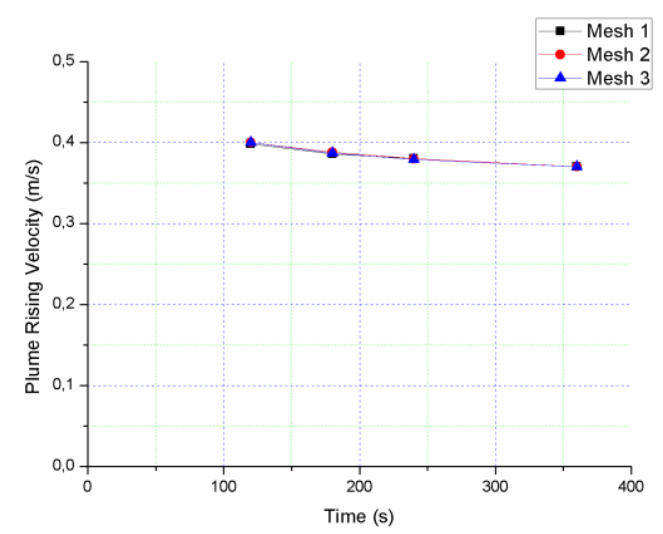

Fig. 11. Mesh independency study: Plume rising velocity comparison

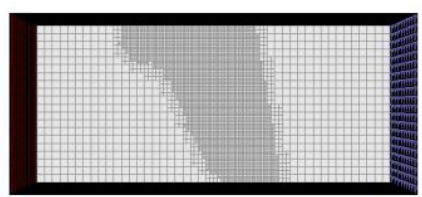

Fig. 12. Characteristic representation of the mesh refinement in the computational domain mid-plane

\section{MATERIALS AND METHODS}

The purpose of the present study is to investigate two cases of oil leak, in the Gulf of Patras, with the presence of gas leaking and transverse sea current, without the use of chemical dispersants and with the use of chemical dispersants, at a dispersant to oil ratio (DOR) of 1:25. The results provide information about:
- The leaking oil rise time to the sea surface.

- The oil bubbles rising velocity.

- The horizontal migration distances during the drifting process, due to the sea current.

The material properties as well as the boundary conditions are based on published data, further ensuring the reliability of the proposed computational model.

The computational domain, as mentioned earlier, is a rectangular region of $130 \mathrm{~m}$ height ( $\mathrm{z}$ axis), $300 \mathrm{~m}$ length (x-axis) and $300 \mathrm{~m}$ width (yaxis), consisting, initially, of 42,320 hexahedral cells. As the gradient adaption curvature method was enabled in order to achieve mesh refinement based on the DPM concentration, the final meshes contained 195,277 and 532,082 for the two examined cases, after $900 \mathrm{~s}$ and $2000 \mathrm{~s}$ flow time respectively.

Assuming a leak of oil and natural gas, with $50 \%$ oil volume fraction, the final oil volume flow rate is $0.11 \mathrm{~m}^{3} / \mathrm{s}$, corresponding to 60,000 barrels per day. After the appropriate modification concerning the presence of gas, in the first case (without chemical dispersants) the oil bubble size was approximated by a Rosin-Rammler distribution, with minimum and maximum diameter of $0.00305 \mathrm{~m}$ and $0.01885 \mathrm{~m}$ respectively, while the mean diameter is $0.0077 \mathrm{~m}$ and the spread parameter is equal to 1.8 [12]. For DOR 1:25 the oil bubble size was approximated by a logarithmic distribution, with minimum and maximum diameter of $0.0003 \mathrm{~m}$ and $0.00219 \mathrm{~m}$ respectively, a mean diameter of $0.00076 \mathrm{~m}$ and a spread parameter of 0.7 [12].

For both cases, diameters correspond to groups of bubbles with volume fraction above $3 \%$ of the total distribution [12], while smaller and greater diameters are neglected. The transverse sea current boundary condition is considered for this initial application, to have a uniform distribution for both cases, equal to $0.1 \mathrm{~m} / \mathrm{s}$ [8].

As in the mesh independency study, the model set up concerning all the other numerical simulation parameters, is the same with the validated one, with the exception of the initial values for turbulent kinetic energy and turbulent dissipation, which were $0.00457 \mathrm{~m}^{2} / \mathrm{s}^{2}$ and $0.0091 \mathrm{~m}^{2} / \mathrm{s}^{3}$ respectively, due to the fact that the seawater is not stationary.

The effects of oil and seawater temperature, as well as the seawater density stratification, were considered negligible for the evolution of the flow, at this stage of research. 


\section{RESULTS AND DISCUSSION}

The distributions of oil bubbles in the computational domain for both cases are presented in Fig. 13 to 16 under the effect of the sea current and the presence of gas, after the first bubbles have reached the sea surface. The oil bubble traces are coloured by bubble diameter. The rise time for the first case (without chemical dispersants) is $351 \mathrm{~s}$ while with DOR 1:25 is $910 \mathrm{~s}$, which corresponds to an increase of $159 \%$.

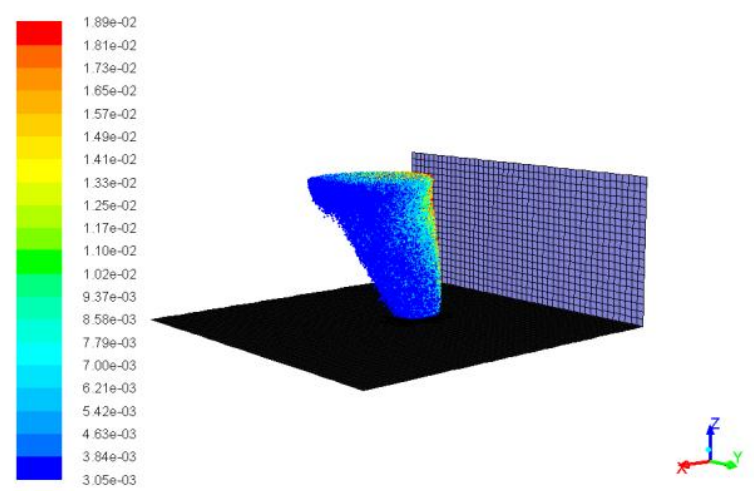

Fig. 13. 3D representation of oil bubble traces coloured by diameter $(m)$, without chemical dispersants
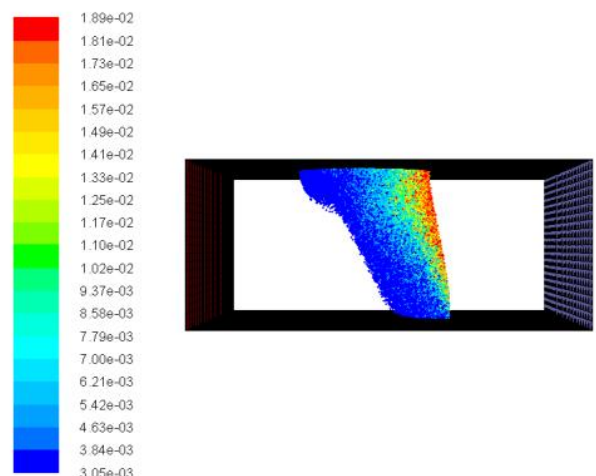

Fig. 14. Oil bubble traces coloured by diameter (m), without chemical dispersants, xz plane view
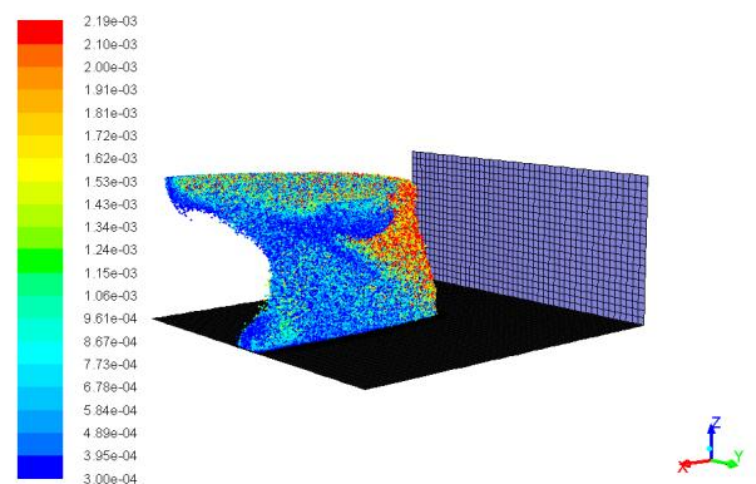

Fig. 15. 3D representation of oil bubble traces coloured by diameter $(\mathrm{m})$, DOR 1:25

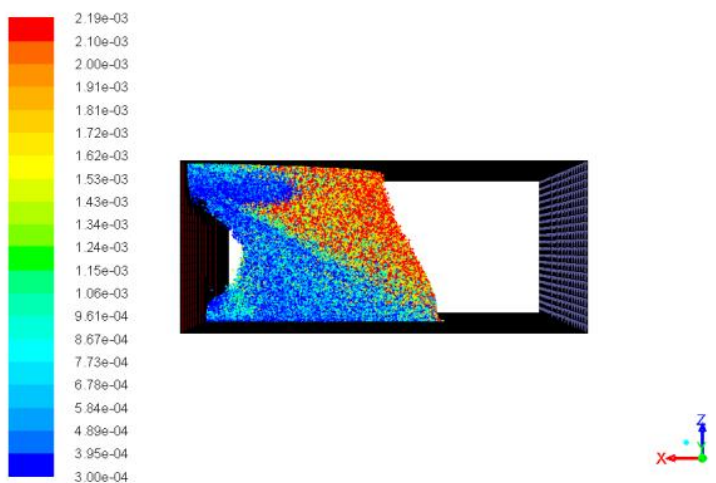

Fig. 16. Oil bubble traces coloured by diameter $(m)$, DOR 1:25, xz plane view

Then, the horizontal motion of the oil bubbles was analyzed, with the results showing that under the effect of the chemical dispersants the bubbles are covering greater distance in the horizontal direction being carried away by the sea current and the corresponding increase of their velocity in this direction. This is evident from the horizontal distance covered at the same instances by oil in both cases, as shown in Fig. 17. After $600 \mathrm{~s}$, the oil bubbles in the crude oil covered $84.7 \mathrm{~m}$ while with DOR 1:25 $94.3 \mathrm{~m}$ and after $910 \mathrm{~s}, 125.4 \mathrm{~m}$ and $136.9 \mathrm{~m}$ respectively.

The horizontal velocity for both cases is slightly decreasing, after the first few seconds of the flow, from $0.17 \mathrm{~m} / \mathrm{s}$ to $0.138 \mathrm{~m} / \mathrm{s}$ for oil without dispersants and from $0.164 \mathrm{~m} / \mathrm{s}$ to $0.15 \mathrm{~m} / \mathrm{s}$ for DOR 1:25, as presented in Fig. 18. Therefore, it is concluded that, in addition to increasing the rise time, the presence of chemical dispersants clearly affects the horizontal movement of the oil bubbles, which are drifted by the sea current, enhancing dispersion, as their smaller diameters lead to less buoyancy.

Another striking effect is the behavior of the smaller oil bubbles in DOR 1:25, with diameters between $0.0003 \mathrm{~m}$ and $0.0008 \mathrm{~m}$, as shown in Fig. 15. Oil bubbles of such small diameter are strongly drifted immediately after their release, towards the current direction resulting to oil droplets significantly dispersed in the water column. This effect is not observed for the crude oil since the greater diameters of oil bubbles offer increased buoyancy and even the smaller oil bubbles cover longer vertical distance along with their horizontal movement. 


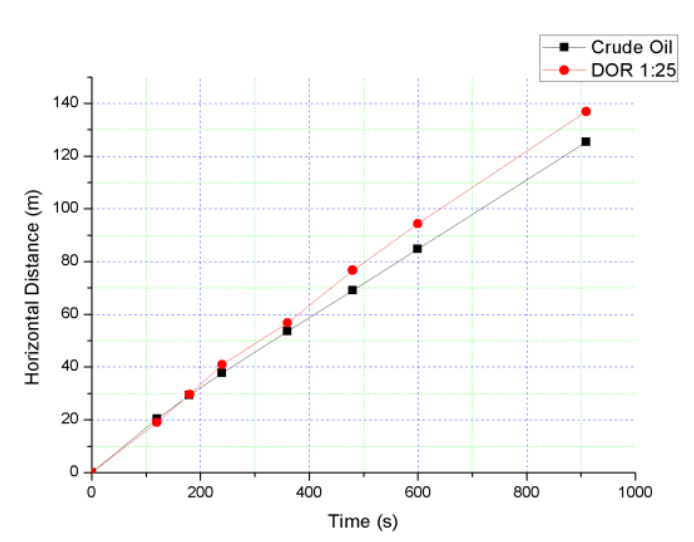

Fig. 17. Horizontal migration distance comparison

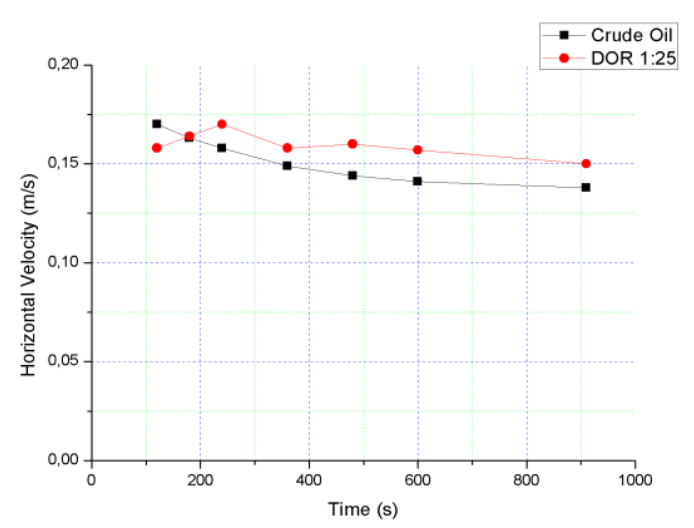

Fig. 18. Bubble horizontal velocity comparison

Fig. 19 demonstrates the severe effect of chemical dispersants on the vertical distance covered by upward moving oil bubbles at several time instances. After $180 \mathrm{~s}$, the crude oil bubbles covered $69.9 \mathrm{~m}$ while in oil bubbles with DOR 1:25, $41.5 \mathrm{~m}$ and after $240 \mathrm{~s}, 91.2 \mathrm{~m}$ and $50.5 \mathrm{~m}$ respectively.

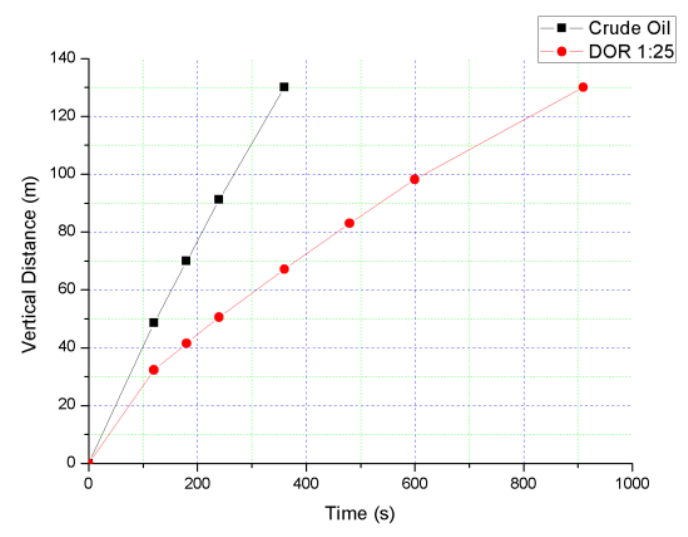

Fig. 19. Rising height comparison

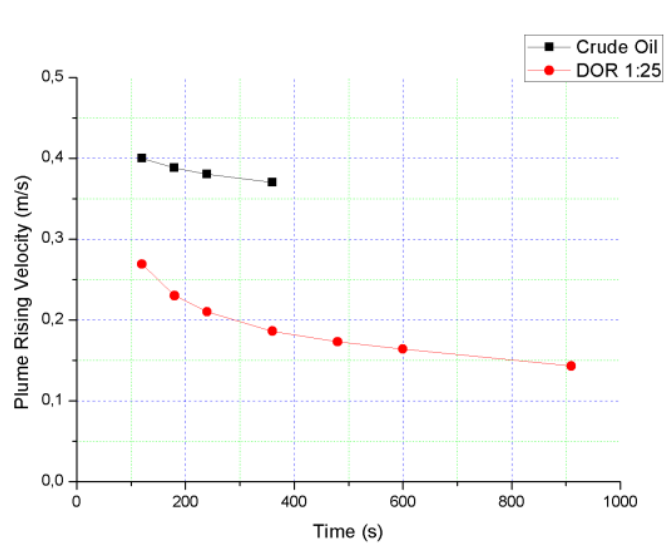

Fig. 20. Plume rising velocity comparison

The evolution of the vertical oil bubbles velocity is presented in Fig. 20. In the initial stage, the velocity is $0.4 \mathrm{~m} / \mathrm{s}$ for the crude oil and $0.269 \mathrm{~m} / \mathrm{s}$ for oil with DOR 1:25. For the case of the crude oil the curve of the plume rising velocity shows a slight decay, with the final value being equal to near $0.36 \mathrm{~m} / \mathrm{s}$. On the other hand for the case of oil with DOR 1:25 the decrease of the plume rising velocity is more rapid up to $350 \mathrm{~s}$ being equal to almost $0.19 \mathrm{~m} / \mathrm{s}$. From this point on the decay rate is slower up to $900 \mathrm{~s}$ where plume rising velocity is equal to $0.15 \mathrm{~m} / \mathrm{s}$. Overall, it is evident that the effect of the chemical dispersants to the rising velocity is decisive, since the significantly less buoyancy is correlated with the reduced oil bubble diameters.

The above data are characteristic of the underwater hydrodynamic behavior of the leaking oil under the effect of transverse sea current and the presence of chemical dispersants. They are useful elements for the organization and implementation of any immediate intervention plan in the area of a possible accident leading to oil-gas leak.

\section{CONCLUSION}

The purpose of the study is to present an easy and effective method, which can represent with sufficient accuracy and in a short time, the situation formed in case of an oil spill, to enable immediate intervention in a limited area, without a large dispersion of forces, providing the possibility for better management. It is observed that:

- The use of chemical dispersants significantly increases the rise time.

- There is an effect on horizontal movement, with an increase in horizontal velocity under the influence of the chemical dispersants, with oil bubbles deflected 
further by the sea current, enhancing their dispersion.

- The movement of oil bubbles is not characterized by sharp velocity changes.

These assessments can contribute to the development of environmental studies that include various accident scenarios as well as to the prioritization of response actions after a real oil leak incident, regarding the proper distribution and utilization of the available resources to deal with it.

\section{REFERENCES}

[1] H. Zhu, P. Lin, Q. Pan, A CFD (Computational Fluid Dynamic) for Oil Leakage from Damaged Submarine Pipeline. Energy, 64, 2014: 887899.

\section{https://doi.org/10.1016/j.energy.2013.10.037}

[2] M. C. Boufadel, F. Gao, L. Zhao, T. Özgömen, R. Miller, T. King, B. Robinson, K. Lee, I. Leifer, Was the Deepwater Horizon Well Discharge Churn Flow? Implications on the Estimation of the Oil Discharge and Droplet Size Distribution. Geophysical Research Letters, 45, 2018: 23962403.

\section{https://doi.org/10.1002/2017GL076606}

[3] M. Bakli, Evaluation of Oil and Gas Dispersion during Subsea Blowouts (Master Thesis). Norwegian University of Science and Technology, Trondheim, 2014.

[4] X. Li, G. Chen, H. Zhu, Modelling and Assessment of Accidental Oil Release from Damaged Subsea Pipelines. Marine Pollution Bulletin, 123 (1-2), 2017: 133-141.

https://doi.org/10.1016/j.marpolbul.2017.09.004

[5] F. Gao, L. Zhao, M. C. Boufadel, T. King, B. Robinson, R. Conmy, R. Miller, Hydrodynamics of Oil Jets without and with Dispersant: Experimental and Numerical Characterization. Applied Ocean Research, 68, 2017: 77-90.

https://doi.org/10.1016/j.apor.2017.08.013

[6] P.E. Makatounis, J. Skancke, E. Florou, A. Stamou, P.J. Brandvik, Management of Oil Spill Contamination in the Gulf of Patras Caused by an Accidental Subsea Blowout. Environmental Pollution, 231, 2017: 578-588. https://doi.org/10.1016/j.envpol.2017.08.076

[7] D.P. Giannoulis, D.P. Margaris, Numerical Simulation of the Three Phase Flow Formed During the Containment of an Oil-Methane Leak Using the DIFIS System. International Review of Mechanical Engineering, 9 (6), 2015: 576-582.

https://doi.org/10.15866/ireme.v9i6.7714

[8] E. Papathanassiou, K. Pagou, E. Krasakopoulou, S. Reizopoulou, A. Pancucci, Strategic Environmental Assessment of the Hydrocarbon Activities for Prospecting, Research and Exploitation within the Licence Area "Western Patraikos Gulf". Hellenic Centre for Marine Research, Attiki, 2012.

[9] Hellenic Hydrocarbon Resources Management, Patraikos Gulf (west) Block, detailed map. https://www.greekhydrocarbons.gr/en/Patrai kosGulf west en.html (Accessed 28.10.2020).

[10] Computational Fluid Dynamics (CFD) PreProcessor, Gambit 2.2.30, 2007.

[11] ANSYS ${ }^{\circledR}$ Academic Research, Release 16.0, available: https://www.ansys.com.

[12] P.J. Brandvik, $\varnothing$. Johansen, U. Farooq, G. Angell, F. Leirvik, Subsurface Oil Releases Experimental Study of Droplet Distributions and Different Dispersant Injection Techniques - Version 2, Final report. SINTEF Materials and Chemistry, Trondheim, 2014.

[13] N. Avgerinos, D.P. Margaris, A threedimensional CFD study of the hydrodynamic behavior of equal and unequal-sized in-line methane bubbles at high pressure. Chinese Journal of Chemical Engineering, 26 (9), 2018: 1792-1802.

https://doi.org/10.1016/i.ciche.2017.12.009

[14] T. Engebretsen, T. Northug, K. Sjøen, T.K. Fanneløp, Surface Flow and Gas Dispersion from a Subsea Release of Natural Gas. The Seventh International Offshore and Polar Engineering Conference, 25 ${ }^{\text {th }}$ May, 1997, Honolulu, Hawaii, USA.

[15] Q. Pan, Modelling of Turbulent Flows with Strong Dispersed Phase-Continuous Fluid Interactions. Norwegian University of Science and Technology, Trondheim, 2014. 